\title{
Jafari and Transformation: A model to enhance short-term overseas study tours
}

\section{Timothy Hall*, Tonia Gray*, Greg Downey ${ }^{\dagger}$, Colin Sheringham*, Benjamin Jones*, Anne Power*, Son Truong*}

* Western Sydney University

${ }^{\dagger}$ Macquarie University

An increasing emphasis on university internationalisation, global citizenship education and outbound mobility experiences (OMEs) has seen international study travel become a key staple of tertiary education. University students undertake learning experience for academic credit overseas for a period shorter than a semester under the guidance of an academic staff member. OMEs may involve student service learning or be tour based and can occur in either single or multiple destinations. In the mid-1980s less than half a million students worldwide spent part of their degree in another country, compared to some three million students in 2011 (Rizvi, 2011, p.693). Australian university students are part of this global trend with participation in OMEs at record numbers. In just three years, the number of students studying abroad increased from 15,058 in 2009, to 24,763 in 2012 (Department of Education, 2014). During the same period, the growth of shortterm OMEs, often less than three weeks, outstripped the traditional semester or year-long exchange. In 2012, OMEs became the major form of outbound mobility with 8,570 Australian students participating (Olsen, 2013, p.14). Dwyer (2004) suggests study abroad programs of at least six weeks are required to maximise learning outcomes. Despite this reservation, the popularity of short-term OMEs continues to grow. In Australia, federal government funding sources, such as the Department of Foreign Affairs and Trade's New Colombo Plan (NCP), provides funding for a range of OMEs, including many short-term study trips.

With student participation increasing, greater consideration needs to be given to the ability of OMEs to deliver high quality learning outcomes to students, particularly within an intensive shortterm format. A majority of existing literature relays the experiences of academic staff in designing and facilitating OMEs (Brokaw, 1996; Gordon \& Smith, 1992; Koernig 2007; Schuster, 1993), but an emerging literature also considers student experiences (Downey, Gothard \& Gray, 2012; Evans, Finch, Toncar \& Reid, 2008; Weaver \& Tucker, 2010). While these evaluations are insightful, the observations may not be relevant to all of the wide variety of OMEs now being offered. This paper promotes a reconstructed Jafari (1987) tourism model as a framework to understand the various components of the student and staff travel experience while participating in an OME, especially short-term overseas study trips. Working through each of the components of the Jafari model allows recognition of the changing needs and requirements of both students and staff throughout an OME and the tensions that accompany this relationship.

To facilitate the discussion we draw on the experience of a 16-day OME to Vietnam offered by Western Sydney University's School of Business. This study tour has been available annually since 
2011. The OME offered to Hospitality Management and Sport Management students involves fifteen undergraduates and two staff (authors on this paper) travelling to four cities covering the length of Vietnam. As part of a for-credit unit, students undertake a series of pre-departure workshops and then participate in a range of overseas field visits, business meetings and cultural activities in pursuit of fulfilling the learning outcome "reflect on an international hospitality/sport environment, including a comparison with your understanding of the domestic hospitality/sport industry". This learning outcome is assessed through the submission of a reflective essay that is completed whilst overseas.

Drawing on experiences gained from the development and implementation of this OME to Vietnam, this paper will demonstrate how a reconstructed Jafari tourism model, explained below, may provide a better understanding of the changing staff and student experience throughout an OME. This framework may be used to better manage expectations and conflicts as well as inform the development of an itinerary with strategically placed activities.

\section{The Jafari Model}

In an influential 1987 article in Tourism Management, Jafar Jafari outlined a tourism model consisting of six integrated components: Corporation, Emancipation, Animation, Repatriation, Incorporation, and Omission. Using a springboard analogy, the Jafari model explores the immersion process that takes place in travel from the ordinary to non-ordinary, and the potentially transformative impact of the return from non-ordinary back to ordinary daily life. He suggests that "tourism is a manifestation of life, an outlook to life, a celebration of life. Yet it is a neglected dimension of life" (Jafari, 1987, p.159). Applied to short-term OMEs, the Jafari model provides intellectual scaffolding to consider how opportunities for both learning and transformation may be maximised, as well as highlighting tensions which develop in the pursuit of these goals. The revision of the model involves placing stronger emphasis upon reflection in each stage of the model sequence.

Given that the Jafari model is nearly thirty years old, the authors acknowledge much has been written on tourism and outbound mobility experiences since. Jafari (2003) himself has traced the evolution of tourism as a subject of scholarly research. A growing body of literature explores the challenges to academic staff of designing and facilitating overseas student experiences (Brokaw, 1996; Gordon \& Smith, 1992; Koernig, 2007; Schuster, 1993). Increasingly, for example, academics debate the most appropriate form of assessment to accompany a short-term study tour (Duke, 2000; Menzies, 2012). While the majority of existing literature focuses on the role of the academic in designing a short-term international study tour, an increased focus on student learning outcomes from study tours encourages us to adopt a complementary student-centred perspective in models of the overseas experience (Brown, 2009; Evans et al., 2008; Gordon, 2009; Gray, Downey \& Gothard, 2012; Weaver \& Tucker, 2010). In light of this new literature, the authors argue that the core principles of the Jafari model have remained intellectually robust and provide an opportunity to consider many of the tensions which may exist between students' and staff's perspectives on OMEs.

Our updated Jafari framework treats short-term OMEs as a hybrid model of travel, combining elements of holiday and adventure with study and education. The hybrid understanding is conceptually important both here and in other forms of travel that straddle the leisure/work divide, 
such as conference travel. Recognising the tension that may exist between staff and student perceptions is important, especially in the motivations and goals for the OME, but our revised model points out that this tension is inherent in the form of travel. The work of Ryan and Deci (2000) is useful here in distinguishing intrinsic from extrinsic motivation. While a student may already be intrinsically motivated to travel overseas and enjoy new experiences, academic staff can foster extrinsic motivation by explaining the many benefits of combining travel with study in an OME. The balance between work and leisure, study and fun, pedagogical and personal outcomes must be continually negotiated both before and during an OME. Applying the six-component model outlined by Jafari (1987) to the travel experience assists us to reconcile potential competing interests and to maximise positive outcomes for both staff and students.

The modified Jafari model contributes three essential pedagogical strengths to short-term OMEs: teaching, planning and reflection values. Teaching value is gained because the sequence offers a simple theoretical framework for students to understand the effects of travel. Once explained, the model is easy to understand and helps demonstrate the links between theory and the students' own experience. Planning value arises from insights into the feelings and actions of both the student and the academic staff at different stages of the trip. The importance Jafari places on empathy is also key to planning OMEs. Understanding that students may take time to adjust to a new culture, organisers may better design OME itineraries to include group-based orientation activities at the start and intervals that allow for emotional adjustment. Finally, reflection-based activities can be refined based on greater recognition of the need for personal growth and mapping the experience. Deep introspection and guided critical self-reflection are vital for encouraging transformative travel. The updated Jafari model acknowledges that the period of reflection involves a transformative change which, once better understood, can be factored in to post-OME sessions for both students and staff.

Our framework encourages a generous approach to transformation and travel. Transformative learning is a well-established academic tradition drawing heavily on the pioneering work of Jack Mezirow in the late 1970s. Mezirow's (1978) ten phases of transformative learning trace the liminal experience from a "disorientating dilemma" and self-examination through to building competence and self-confidence and reintegration with a new perspective. This process is the basis of long-term transformation resulting in a fundamental paradigm shift. Transformation can also be short-term and situational: students may display different behaviour and even attitudes in response to a dramatic change of environment.

According to Gray and colleagues (2012), the transformative power of an international sojourn is not inevitable without scaffolding and pedagogical support. Other researchers (such as Brown, 2009; Brunner, 1991; Lathrop, 1999; Lean, 2009 \& 2011; Neppel, 2005; Raymondi, 2005; Stitsworth, 1987; and Yachimowicz, 1987) have highlighted the transformative potential of travel. But, as Bruner (1991) cautions, students often describe this process of change in stereotypical forms, such as claiming that the OME was "a trip to remember" or "a trip of a lifetime". Those facilitating OMEs often hear generic statements in assessment from newly returned students such as, "I went away a girl/boy and came back a woman/man" or "I've learned how fortunate I am to be born in Australia". These sorts of stereotypical narratives erode the potential for deeper change and growing awareness. The challenge for those leading OMEs is to find innovative ways of "teasing out the 
processes that underpin these types of statements" (Gray et al., 2012, p.5) in order to facilitate the on-going critical self-reflexivity that supports the development of new perspectives or even worldviews.

Our version of the Jafari model incorporates both short and long-term transformation and provides academic staff with a framework for both monitoring and fostering student growth. During animation component of the model, students are likely to take on new roles: that of the tourist or even the ambassador of their home country. While this form of transformation is temporary, the broader experience, especially for students who may not have travelled before, can inspire self-examination and longer-term change. As Lean, Staiff and Waterton contend, travel is not simply a shift of location, but a journey that is experienced "physically, virtually, imaginatively and communicatively" (2014, p.12). Through guided reflection and institutional support, the opportunity for positive transformation can be channelled into more profound gains, away from superficial or stereotypical self-understandings. For example, students can be encouraged to reflect much more on what their hosts experienced, or how the students themselves might have been perceived by those that they visited, rather than focusing exclusively on their own experiences or sense of achievement.

What constitutes short-term student mobility varies across institutions and among programs, with variables such as multiple city (Gordon \& Smith, 1992; Menzies, 2012; Schuster, 1993) or single city trips (Brokaw, 1996). Programs exist for undergraduate students (Koernig, 2007), postgraduate students (Schuster, 1993), and for tours involving both levels of university students (Hutchings, Jackson \& McEllister, 2002). Design varies significantly depending upon the region of the world travelled, with Europe being a frequent destination for American-based study tours (Gordon \& Smith, 1992; Schuster, 1993; Porth 1997), and Central America (McKenzie, Lopez \& Bowes, 2010) and Asia for Australian-based universities (Hutchings, Jackson \& McEllister, 2002; Menzies 2012; Weaver \& Tucker, 2010). Regardless of this variation, the following six components of the Jafari model offer a framework for consolidating the learning and transformational potential of OMEs while also becoming aware of inherent tensions caused by the nature of these educational trips.

\section{Corporation}

Every journey involves movement, but a decision must first be made to step willingly away from the ordinary and towards the non-ordinary. We must choose to climb onto the springboard before we can leap. The corporation component of the model consists of two phases, the first of which considers the range of factors which motivate the individual to want to be a tourist. In response to this, the OME to Vietnam includes a pre-departure reflection on why the university student chooses to undertake the journey, what their expectations are, what they look forward to and what they might fear. Through this process, the individual arrives at the second phase of this corporation component in which the decision to travel is made. In this section we consider corporation through the eyes of both staff and students.

Crucially, we must recognise that the intrinsic motivations of the staff and students will overlap but will likely not be identical. A desire to visit popular and culturally prominent destinations often informs students' motivations to travel. From these motives, a tension may emerge between the ideals of a holiday and those of a study trip. Students' perceptions of a destination also have an impact of the marketability of some OMEs. For Australian students, Vietnam is not necessarily a 
first-choice destination, but from a university and government perspective, the country has many merits. For example, the 'Advice to Applicants' for the New Colombo Plan specially encourages increased student mobility to the Indo-Pacific region. To counter this gap in motives, staff need to put considerable effort into highlighting for students the desirability of destinations like Vietnam for study travel, building familiarity with these destinations by regularly using them as examples in teaching and references to previous trips.

As a hybrid form of travel, a university OME must have tangible benefits that outweigh potential costs. A student could, of course, visit Vietnam on his or her own, purely as a tourist. The advantage of a short-term OME is that, although students can enjoy the touristic aspect of the trip, they are also guided towards deeper learning that can carry through beyond graduation, with outcomes like increased employability. The advantages, such as distinctive cultural access or gaining valuable familiarity with working in an international setting, must be clearly and regularly communicated to bridge this gap in corporation experience.

In the lead-up to an OME, students will participate in their life and be influenced by a range of factors that will impact their overall desire to travel. In the case of the Vietnam OME, academic staff actively encourage students to move to the second phase to generate interest in the OME to provide a meaningful experience for the students but also to meet the staff's own work-related agenda. Jafari (1987) refers to a tourist market; in this regard, students are the OME "market". A coordinated series of emails with links to previous staff and student blogs, itineraries and answers to frequently asked questions are supported by in-class information sessions in the semester prior to the OME. From a "marketing" or corporation perspective, staff must balance the information they provide to arouse student interest with the risk of providing so much information that the sense of mystery and stepping into the unknown is compromised.

For the staff member, the corporation component of the model is significantly different. Planning an OME equates to work and organisation. Preparation can be time consuming and may start almost a year in advance. As a result, the corporation for staff is likely to start well before that of the students. Some of the planning and logistical processes required for an OME may include seeking approval, securing funding and institutional support, and then selecting staff, which may occur through nomination or a competitive process (McKenzie, Lopez \& Bowes, 2010). Academics will consider a range of factors in deciding whether to undertake the travel and responsibility for the welfare of students. Once tour leaders take on responsibility for an OME, the process of organising activities becomes a balancing act between educational and leisure elements, practical constraints and pedagogical ambitions. Organisers will be challenged by the need to schedule meetings with government representatives and organise appointments with business leaders, visiting universities or teaching classes whilst trying to balance the desire of students for free time or touristic outings. The selection of OME activities may also need to find a balance between experiences that provide quality outcomes for the students against boredom for staff who may be repeating activities if they have previously participated in the OME.

In the case study example, some students decided early in their degree program that they would participate in the Vietnam OME, whilst others took longer to decide. Once presented with the opportunity to participate in an OME, students may begin considering aspects identified by Evans 
and colleagues (2008) such as: How much will it cost? Where is the study tour going? Are my friends going? How much leisure time is involved? The decision to participate in an OME may involve a struggle to balance influences of family and friends, financial considerations (the cost of OME as well as personal spending money), and work and family commitments. Students may also be caught between competing desires that are not obvious to organisers. For example, students previously expressed a desire to attend music festivals which conflicted with OME dates, which the organisers did not realise. Previous travel experiences may influence students in this component; prior travel experience within any OME cohort may vary significantly. For example, in the 2014 OME to Vietnam, one student had migrated as an adult from England whereas some of the other students needed to obtain passports for the first time for the purpose of the trip.

\section{Emancipation}

The emancipation component of this model involves symbolic separation through a series of spatial markers, each of which represents a removal from the ordinary. This early component is vital in the process of transformation, although it starts with de-individualization because travel removes one from familiar contexts. Jafari notes that, "while away from home, the real identity of the traveller is disguised. His own face becomes an anonymous mask behind which he hides" (1987, p.153). The revelation that the traveller will soon be far removed from the regular structures that govern ordinary existence prompts an excited energy described as "emancipative magic" (p.153). Academically, we can recognise the importance of the separation process and take steps to help mark the transformation. Even something as simple as a photograph of the group leaving behind the ordinary, public area of the airport and proceeding into the restricted Customs area can provide a useful symbolic marker. Jafari highlights two essential phases within emancipation: 'separation' or the growing distance from the ordinary, and 'declaration,' the shrinking distance to the non-ordinary.

For some students, the sensation of emancipation may begin while driving to an airport. For others boarding the plane or arrival at the destination may be felt as the point of demarcation. Once at the destination, the students will contribute to a declaration process when they begin to undertake activities that separate them from their quotidian habits; activities such as navigating motor scooterfilled roads or cashing foreign currency may highlight the different texture of even banal moments. In the models and literature reviewed, this component seems to be overlooked, yet emancipation forms an important threshold, especially for those students who have not previously travelled without the security of family and friends. For many of these students, this component will involve a farewell to family or friends at home or at the airport, which may accentuate the impact of spatial markers.

Students of differing levels of travel experience will pass through the emancipation component at different rates. Tour leaders need to recognise that, as a result, some students still may be struggling with elements of separation and associated home sickness whilst others declare their arrival as tourists early and easily. Students who more quickly and seamlessly enter the declaration phase of emancipation may assist other students with separation issues and lead by example. While Jafari (1987) focuses on the experience of the individual, the ability to move through emancipation may also occur as a function of the group. In fact, the group itself may move through the emancipation component collectively, which staff members and activities can facilitate. For example, Koernig (2007, p.213) uses a first-day group walk and riding on public transport as a way to 
acclimatise to the local environment. Familiarity with the local area and an ability to use transport provides students with an increased level of confidence that can assist their transition. The design of an OME itinerary has the potential to impede or to facilitate students passing through emancipation.

Using this idea from Koernig (2007), the Vietnam OME tries to facilitate students moving through the emancipation component of the model by starting day two with a half-day city tour of Ho Chi Minh City; the activity allowed students to orient themselves and observe their new environment from the perceived safety of a bus. This outing presents students with the opportunity to observe the traffic, city, and people, whilst also providing a chance to ask questions of tour guides to help settle nerves. The tour involves a need for students to exchange money and take out their cameras as part of the process of moving into declaration. During the half-day city outing, students are required to wear a tour shirt that has the university name and logo on the left side of the chest. The aim of the tour shirt is to generate a sense of security within the group as it practically makes head counts by tour guides and staff easier. At a deeper level, the shirts are symbolic of the springboard away from the ordinary world of student work while fostering a sense of cohesion and identity. The badging of the shirts provides a visual reference to the cultural baggage carried. The half-day tour finishes at a local market and gives the students the option of staying - pairs or small groups encouraged - to allow them to buy souvenirs such as the traditional conical hat. The popular piece of memorabilia is rich with cultural meaning and juxtaposes sharply with the tour shirt. Staff should be aware of their capacity to facilitate learning during the declaration phase. In 2013, the majority of students on the Vietnam trip purchased a traditional conical hat as a souvenir. However, in 2014, after a staff member in passing noted to the group the difficulty of packing conical hats, none of the group purchased the souvenir. The comment was innocent, but on reflection one that could be seen as value-laden.

When they finished shopping, students practiced how to cross the road by themselves. This simple activity requires a distinctive skill set in Vietnam that is unnecessary in Australia due to the prevalence of motor bikes, relative scarcity of pedestrian crossings, and lax traffic rules and protocols. The activity finishes at the markets around lunchtime with the next activity to commence in the hotel foyer in early evening. The aim of the scheduling is to provide enough time to let students explore for a short period without being overwhelmed. By appreciating the components of the Jafari model, the first day in country of the OME itinerary assists students through the emancipation component and provides a balance of orientation and education.

For the staff members, the emancipation likely takes a different form. The period involves immediate practical responsibilities, such as numerous head counts, organising various modes of transportation, fielding questions from students (and loved ones) and being empathetic to a range of emotions, ranging from fear through to excitement. Separation will consist of a change in physical environment, while declaration is less likely to take the form of tourist garb but will no doubt include administration concerns. From practitioner's experience, the change is heightened by the process of saying goodbye to family and moving into secured areas of the airport. This physical movement can be seen as a metaphor of the transfer of responsibility from parents and loved ones to academic staff. For the academic, the declaration is often symbolised by an increased sense of 
responsibility and feeling of isolation given that they are the point of contact for a group of students in a foreign country and may not speak the local language or languages.

Given that generational differences will separate staff and students, their transitions through the emancipation component of the Jafari (1987) model will likely be divergent. One academic from the Vietnam OME suggested that the emancipation component of the model has two distinct phases. The first is when group leaders start to get the bag ready a few days before departure and make sure the first aid kit is up to date, confirm the first few in-country activities with industry partners, finalise arrangements with travel agents, and respond to student last-minute enquiries. The second involves ensuring people at home are prepared for your absence; tour leaders must rush to finish last minute practical chores at home while also preparing family and friends for one's absence. For example, we felt the need to do something special with the kids before an absence of two weeks.

\section{Animation}

Arrival in the travel destination begins a new component where the culture of the non-ordinary is dominant. At this point the transformation process intensifies, and the traveller enters the "tourist trance" (Jafari, 1987, p.153). Within animation, "the tourist further internalizes the idea that he is no longer imprisoned by his former self but is transformed into a new person with a new identity" (Jafari, 1987, p.153). This component is characterised by a sense of both freedom and fantasy. For students on OMEs, a unique opportunity is presented not only to play a different part, but also to act in a different context to the ordinary. With this freedom comes a tension between staff and students in that some of the activities identified by Jafari (1987) as potentially part of the animation component may be in conflict with standards for acceptable behaviour whilst representing the university in an international setting. In some host countries there may be a prevalence of bars and nightclubs that form part of the night time economy and are of interest to students. However supervising staff may impose alcohol consumption restrictions and curfews in an attempt to avoid any alcohol related incidents that may not be well received by the hierarchy of their university.

The animation component of the model sees students undergo a period of orientation to their new surrounds, and the experience will inevitably vary based on previous travel experience and familiarity with the destination. As time progresses, students will gain confidence and become immersed in the travel experience. Such an immersion may be gradual and may see cultural norms of the home ignored. While the students take on a form of temporary transformation by becoming a tourist, the seeds of longer-term transformation are also being sowed. Illeris (2014) notes that identity is structured in three primary layers: core identity, personality layer, and preference layer. Even a short-term OME has the ability to affect all three as the student gains (or expands) a new aspect of their identity; they are an international traveller.

The progression of students during the Vietnam experience to animation was often manifested in small but significant ways such as displaying competency interacting with local people, shopping and getting around. Eventually animation enters a valediction phase in which the tourist participates in the last of the tour activities which have removed them from everyday life (Jafari, 1987). Brokaw's (1996) single city model of study trips would allow students to more fully embrace the animation component and develop a deeper sense of place; however, with this level of comfort comes the risk that students may become over confident with their surroundings. As confidence builds students 
participating on the Vietnam OME become more interested in the timing of organised activities as they try to maximise their period of valediction. With this growing confidence comes a reluctance to wear a tour shirt that symbolically marks them as being from overseas, impeding their ability to participate in life in their new context on their own terms.

Conscious of this student transition, academic staff may use physical reminders to temper the move into valediction. This physical representation of the tour shirt also demonstrates growth as the same tour shirt brought a sense of comfort during the emancipation that has long passed. Another way in which the transition to valediction can be managed is through the schedule and timing of activities within an itinerary. According to Koernig (2007), a two-week OME can be split as follows: 40 percent for company tours, 40 percent structured cultural activities, and 20 percent free time (with a majority of these fitting within the animation component). The structured activities provide a level of surety and control for the academic staff and students, whereas the 20 percent free time provides an element of risk and unknown. The role for the academic is to facilitate a natural learning progression towards familiarity in an unknown place and culture, and towards becoming more globally minded. As Ibrahim (2012) writes, global citizenship embraces an awareness of the particularity of 'my language, culture or heritage' as well as expanding to acknowledge and respect the culture of others (p.14). The tension is that academic staff facilitates exploration and experience of a different place and culture, but at the same time, must be aware of the consequences of overstepping both their competence and local norms for behaviour.

In Vietnam, we managed this tension to a certain degree by providing an itinerary which was initially highly structured with scheduled activities in both the day and evenings in order to minimise students' opportunities to become fully immersed in valediction. However, this pace cannot be maintained for the full two weeks - nor should it be. Part way through the trip, the itinerary provides a twenty-four hour rest period across two days in which students are given the option to put on their tourist mask and embrace the "tourist trance". Without actively structuring this opportunity for valediction, the timing of the rest period on the tour, the city in which it occurs, and the scheduled activities around the period allow some degree of control of the animation component of the model.

The animation component can be physically and emotionally exhausting for academic staff as they assist students to become acclimatised to their new surroundings. However, once animation is embraced, staff may still have an element of practical head counting and organising activities and schedules. For this reason staff should share the leadership responsibilities so they are also able to allocate 20 percent of the tour to free time, this may be achieved through staff filling the roles of tour leader and leader in training. For staff who have previously travelled to the destination, progression through the orientation phase may be quicker. But animation for staff may be stop and start in nature as tour activities may require a shift in roles. During free time for staff, however limited, animation can occur although it tends to be short and ranges from a quiet stroll in the markets through to in-depth, targeted cultural experiences related to personal interests. Animation may be impeded by the fact that academic staff may be required to participate in normal work duties through email and phone communications. Another layer of complication is that difference in time zones and the need to be available for normal work duties may impact academic staff's ability to 
immerse in the animation component as they may be engaged with responsibilities at their home institutions at odd hours.

For some students (and staff) the animation can be confronting. The non-ordinary will involve coping with the rigours of travel, new cultural norms, new food and new friends. For many, the novelty becomes overwhelming which manifests itself in the form of homesickness. The animation component more generally is fraught with tension due to the multi-layered nature of an OME. This stress is not simply a function of the study tour itself but also a function of globalisation, technology, social media, and the degree to which students inhabit the digital world. While physically separated from the ordinary world of home, depending on the destination context, the student on tour will often be surrounded by the familiar (such as global brands), be in very frequent contact with home through technology such as Skype, and may not leave their ordinary digital milieu (Facebook continues). In many ways, the proliferation of social media serves to exaggerate the animation component. Student photos and comments sent to their digital networks often highlight the extent to which they have engaged in a different culture. Images of students in local dress, eating exotic foods, and observing different cultural practices are particularly popular. Digitisation of the travel experience allows the animation process to be broadcast live to a virtual audience. The revised Jafari model is affected by the globalisation of the ordinary. As a conceptual framework, the component remains valid, but the impact of immersion into tourist culture is potentially changed through easy online access to the ordinary world. The technological tether may hold students back from complete immersion.

\section{Repatriation}

In the final days of an OME, the realisation will set in that the animation is nearly complete, and the transition from non-ordinary to ordinary will soon begin. For both staff and student the repatriation component may come through contemplation that the scheduled activities are coming to an end; the close cohort of fellow travellers will soon disperse and be replaced by the familiar faces of home. The repatriation component includes phases of submission in which participants acknowledge that the tourist experience is over and that, by the time the plane touches down, aspects of everyday life will begin to once again dominate their thoughts. This "psychological retransformation" is accompanied by a "resurrection of the former self" and a "yielding to the ordinary world" (Jafari, 1987, p.155). For students on the Vietnam OME, this return can be an emotional one as students work on completing a reflective piece of assessment. While the former self is being revived, it will not be exactly the same, as the OME experience will leave a cultural fingerprint, which varies across each individual, on the traveller. Students need to be given the opportunity to examine and articulate the ways in which they have been affected in both formal and informal settings.

Staff and students will find their own ways of saying goodbye; time should be given for this process. Informal repatriation may include activities like last-minute shopping, especially to pick out souvenirs to keep as a reminder of the experience and to give to family and friends in a symbolic marriage between the non-ordinary and ordinary. The repatriation process may include taking photos and exchanging contact details with both new friends and fellow travellers, or returning to favoured attractions or activities one last time. A formal setting could include a final group activity or an open forum to discuss the trip. Closs and Antonello (2011) have highlighted the importance 
of self-reflection in the learning process and its potential as a conduit to transformation. In Vietnam, the group participates in an extravagant final group meal which in some ways symbolises the farewell to friends and country. A formal mode of repatriation can offer a chance for student reflection on their experience and allow the group to channel their emotions and deal with the inevitable submission to the ordinary together. Upon return to their home country, a symbolic transfer of responsibility occurs from academic staff back to parents, friends and loved ones who greet students at the airport upon arrival.

\section{Incorporation}

While the tourist experience will inevitably come to an end, the incorporation component of the model sees elements of the experience woven into everyday life. Incorporation could occur through a change in behaviour or perception, or the inclusion of artefacts into the everyday. The importance of including a post-travel review or debrief in which students and staff reconnect is discussed by Porth (1997) who identifies this process as an important way to solicit feedback, conduct presentations and draw linkages between theory and the study tour activities. McKenzie, Lopez and Bowes (2010) highlight the need to undertake a post-trip evaluation and student reflection. Outside of these reflections, we have little insight into the longer-term impact of the international study tour on students.

Incorporation resulting from OME is not particularly well understood from a student perspective. Research by Weaver and Tucker (2010) seeks to address this gap by interviewing students twelve to eighteen months after they return from short study tours. Their research makes evident that no definitive characteristic of transformation occurs within the students. Scope for this type of research expands to the tours and may also delve further into how the experience becomes incorporated into everyday life. In the case of the Vietnam OME, some students obtained employment from their direct involvement in the tour, whereas other students were not heard from again.

The level of incorporation may be related to the degree that students enjoyed the OME. Some students shop for local mementos of their trip which may be kept on display, while others take up the suggestion of academic staff to include the OME experience in their resume. The variation highlights the need for the same level of preparation to go into post-tour sessions as into the planning of the actual trip. Whilst elements of the study tour provide a significant number of unknowns, equally, post-tour sessions may also present unknowns concerning the emotional impact of the OME on students. For staff, the level of incorporation may vary with the requirement to report back to the university on the trip, balance expense accounts, and review the tour design for potential improvements and changes to future OMEs. This desire and need to change itineraries for future study trips provides a link to corporation for subsequent sojourns.

\section{Omission}

The omission component acknowledges that whilst tourists are away, elements of their ordinary lives continue without them. An absence from work does not mean that a business stops working, and family members not participating in the tourist experience continue with their everyday lives. This missing part of the everyday may lead to a culture shock when travellers return to everyday life. However, some thirty years after the model was initially proposed by Jafari, the omission component 
has changed with the development and use of information technology and social media. Both students and staff are able to maintain contact with home and work through a range of technologies, including face-to-face communication through tools such as Skype and updates on what friends are doing through Facebook, regardless of distance or time differences. All of these channels have reduced the intensity of omission to some extent. The element that is still prevalent though is culture shock and how the change in cultural setting may have an impact on both staff and students

\section{Conclusion}

This paper analyses OMEs undertaken in Vietnam to provide a case study example to propose a revision of the Jafari model. The new framework is applicable beyond the Australian-Vietnamese context. The Jafari model is a useful tool that provides insight and a framework for understanding staff and student travel experience and transformations associated with OMEs. With its close examination of the transformative travel experience, the updated Jafari model offers pedagogical scaffolding to maximise learning outcomes and cultural exchange for students. The framework offers a holistic approach to travel by illuminating, not only the standard three phases of travel (predeparture preparation, on tour and integration of learning), but the intermediary components that mark the journey away from the familiar.

The gap between staff and student experiences are readily apparent at each component of the model. The six integrated components can serve universities and other academic institutions when designing a short-term OME that promotes positive transformation to anticipate recurring issues and better design complementary programming. The intricate relationship of the ordinary and nonordinary offers guidance when organising student activities not only while on tour but also predeparture and post-return as well. While the traditional year- or semester-long OME may generally have a more lasting impact, the Jafari model can help structure short-term trips to be as conducive as possible to transformation.

Through the lens of our adaptation of Jafari's (1987) model, the inherent tensions of an OME are more clearly understood. The short-term nature of some OMEs requires a balance between academic, cultural and leisure activities; they will constantly compete with each other during the intensive tour timeframe. The model provides a valuable template for labelling aspects of the tourist journey. On top of this, the identification of behaviours, motivations and emotions has proved useful in terms of scheduling, planning and structuring activities at all aspects of OMEs to better manage many of these perceived tensions. The OME is a hybrid approach to travel that combines elements of study, with potential for career development, and leisure. For this reason, the Jafari model is particularly valuable for mapping the complex experience of transformative travel while assisting the organisers of short term OMEs.

\section{References}

Brokaw, S. C. (1996). Planning, organizing and executing short term international exposures for U.S. students of marketing and business: An alternative method. Marketing Education Review, 6(3), 8793.

Brown, L. (2009). The Transformative Power of the International Sojourn: An Ethnographic Study of the International Student Experience. Annals of Tourism Research, 36(3), 502-521. 
Bruner, E. (1991). Transformation of self in tourism. Annals of Tourism Research, 18, 238-250.

Closs, L., \& Antonello, C.S. (2011). Transformative Learning: Integrating Critical Reflection into Management Education. Journal of Transformative Education, 9(2), 63-88.

Department of Education. (2014). Outgoing international mobility of Australian university students. Research Snapshot, July, 1.

Downey, G., Gothard, J. \& Gray, T. (2012.) Bringing the Learning Home: Re-entry programs to enbance study abroad outcomes in Australian Universities. Sydney: Office of Learning and Teaching.

Duke, C. R. (2000). Study abroad learning activities: A synthesis and comparison. Journal of Marketing Education, 22(2), 155-165.

Dwyer, M. (2004). More is better: The impact of study abroad program duration. Frontiers: The Interdisciplinary Journal of Study Abroad, 10, 151-163.

Evans, J., Finch, J., Toncar, M.F., \& Reid, J.S. (2008). Student Perceptions of and Preferences for a Short Overseas Study Tour. Contemporary Issues in Education Research, 1(3), 11-17.

Gordon, P., \& Smith, D. K. (1992). Planning, organizing and executing short term international exposures for U.S. students of marketing and business. Marketing Education Review, 2, 47-53.

Gordon, R. (2009). Going Abroad: Traveling Like an Anthropologist. Boulder: Paradigm Publishers.

Gray, T., Downey, G., \& Gothard, J., (2012). Bringing the Learning Home: Transformation: A resource for study abroad. Transformation Instructors Module. Sydney: Australian Learning and Teaching Council.

Hutchings, K., Jackson, P., \& McEllister, R. (2002). Exploiting the links between theory and practice: Developing students' cross-cultural understanding through an international study tour to China. Higher Education Research \& Development, 21(1), 55-71.

Ibrahim, B. (2012). International service learning as a path to global citizenship. pp. 11-21 In J. Hatcher \& R. Bringle (Eds.) Understanding Service Learning and Community Engagement. Charlotte, NC: Information Age Publishing.

Illeris, K. (2014). Transformative Learning and Identity. Journal of Transformative Education. 12(2), 148163.

Jafari, J. (1987). Tourism models: The sociocultural aspects. Tourism Management, 8(1), 151-159.

Jafari, J. (2003). Research and Scholarship: The basis of tourism education. The Journal of Tourism Studies, 14(1), 6-16.

Koernig. S. (2007). Planning, Organizing, and Conducting a 2-Week Study Abroad Trip for Undergraduate Students: Guidelines for First-Time Faculty. Journal of Marketing Education, 29(3), 210-217.

Lathrop, B.J. (1999). The Influence of Study Abroad Programs on United States Students' Psychological Development. PhD dissertation. University of Georgia, United States.

Lean, G. (2009). Transformative Travel: Inspiring Sustainability. In R. Bushell \&

P. J. Sheldon (Eds.) Wellness and Tourism: Mind, Body, Spirit, Place. Elmsford, NY: Cognizant Communication.

Lean, G. (2011). Transformative Travel: The socially mobile de/construction of reality. $\mathrm{PhD}$ dissertation: University of Western Sydney.

Lean, G., Staiff, R., \& Waterton, E. (2014). Travel and Transformation. Ashgate: London.

McKenzie, R., Lopez, T., \& Bowes, D. (2010). Providing International Opportunities For Business Students: A Guide to Planning a Short-Term Study Abroad Program at Regional and Small Universities. American Journal of Business Education, 3(8), 59-66.

Menzies, J.L. (2012). West meets East: Examining the use of reflective journals in international study programs. Journal of International Business Education, 7, $121-140$.

Mezirow, J. (1978). Education for perspective transformation: Women's re-entry programs in community colleges. New York: Teacher's College, Columbia University. 
Neppel, J. (2005). Study Abroad as a Passport to Student Learning: Does the Duration of the Study Abroad Program Matter? MA dissertation. University of Maryland, United States.

Olsen, A. (2013). Research Agenda: Australian Universities International Directors' Forum. Australian International Education Conference, 9 October.

Porth, S. J. (1997). Management education goes international: A model for designing and teaching a study tour course. Journal of Management Education, 21(2), 190-199.

Raymondi, M. (2005). Latino Students Explore Racial and Ethnic Identity in a Global Context. EdD dissertation. State University of New York at Binghamton, United States.

Rizvi, F. (2011). Theorizing student mobility in an era of globalization. Teachers and Teaching: theory and practice, 17(6), 693-701.

Ryan, R. \& Deci, E. (2000). Intrinsic and Extrinsic Motivations: Classic Definitions and New Directions. Contemporary Educational Psychology, 25, 54-67.

Schuster, C. P. (1993). Planning and implementing overseas travel classes for executive MBA students. Marketing Education Review, 3, 54-60.

Schuster, C.P. (1993). Planning and implementing overseas travel classes for executive MBA students. Marketing Education Review, 3 (Fall), 54-60.

Smith, Waterton \& Watson (2012). The Cultural Moment in Tourism: Moments, Instances and Experience. Routledge: London.

Stitsworth, M. (1987). Personality Changes of American Teenage Participants in a Japanese Youth Exchange. EdD dissertation. Indiana University, United States.

Weaver D. \& Tucker, M. (2010). An international business study tour: A student perspective. Research and Development in Higher Education: Reshaping Higher Education, Proceedings of the 33rd HERDSA Annual Conference, Deakin University, Melbourne, 6-9 July.

Yachimowicz, D. J. (1987). The Effects of Study Abroad during College on International Understanding, and Attitudes toward the Homeland and Other Cultures. $\mathrm{PhD}$ dissertation. University of California, United States. 